The intervention proved overall positive effects on severity of HD symptoms, supporting the benefits of skin care in the workplace. The fact that the intervention showed a larger effect in a subgroup with mild symptoms suggests that this intervention might be of particular importance for primary prevention.

\section{B.6 OCCUPATIONAL CONTACT DERMATITIS AND HOW THE COMMUNITY RESPONSES IN DEVELOPING COUNTRY}

Shambhu Joshi*. Far Western Community Hospital, Kailali, Nepal

\subsection{6/OEM-2019-EPI.121}

Background Dermatologic disorders generally have a major impact on patients daily activities, psychological and emotional state and social relationships in a resource poor setting. Country with different climate zones and environment as well as traditional use of herbal and increasing use of pesticides contact dermatitis is one of the important occupational hazards in construction workers, nearby people and it often leads to poor quality of life of the workers with substantial financial loss.

Methods This study was conducted in three different geographic population. Free health camp was organized with total 310 cases of dermatologic problems were registered in Skin OPD last two years. Patients includes children to geriatric population both male and female groups.

Results Out of the total $42 \%$ were male and $58 \%$ were female patients. Contact dermatitis were found among 28\% of the patients, age and sex had no significant role on prevalence of Contactdermatitis. Urban area patients have tendency due to hair dye, industrial cement and detergents, however rural area patients have causation due to herbal plants (Parthenium dermatitis) and traditional occupational works. Both have nickel, atopic and unknown cause. Foot was the most commonly involved site $(25.7 \%$ of patients). Atopy was present in 18 patients $(25.7 \%)$. A total of 22 irritant reactions were noted in 13 patients. Most of the patients neglect the contact dermatitis due to lack of health information and use of irrational medication also seen.

Conclusion Most of the contact dermatitis is preventative and manageable in time. Due to Climate, geographical areas, literacy difference community preventive health program plays important role, it. Prick-Patch testing, Teledermatology and Geographical information system is another future perspective for dermatology disease mapping in Nepal so that the government can make essential policy, programs for communities so that we can prevent and treat the contact-dermatitis on time.

\section{Mini-Symposium 3: Military Epidemiology}

\section{C.1 THE NZ VIETNAM VETERAN FAMILY STUDY: A MULTI- GENERATIONAL PERSPECTIVE ON HEALTH AND WELLBEING}

David McBride*, Amy Richardson, Brian Cox. University of Otage, Dunedin, New Zealand

\subsection{6/OEM-2019-EPI.122}

Background The serious impact of Vietnam service on New Zealand veterans arose from high levels of combat experience and exposure to chemical agents including Agent Orange. In 2009, we assembled a cohort of 2783 men and 23 women Vietnam veterans. They had significantly increased risks of head and neck cancer and chronic lymphatic leukaemia, with high rates of hospitalisation for renal failure and drug and alcohol problems. Overseas data indicates that families have been affected by relationship difficulties, with subsequent risks of adverse psychological outcomes in children. There have been few longitudinal studies which include a comprehensive exposure assessment (the exposome) and include both veteran and family, which is what we propose.

Study population Using the cohort data, the electoral roll and an information campaign, we will recruit veterans, their spouses and their children for follow up. For all groups, we will obtain data to inform the general exposome through a base-line health survey using some of the brief measures deployed in our 'contemporary veterans' study, including the Post Traumatic Checklist (Civilian or military) for PTSD, and the Brief Family Relationship Scale exposure history.

The specific exposome, including chemicals and 'other' specific exposures, will be recorded by on-line questionnaire and subsequent interview.

Effects will be assessed through linkage to the Birth Defects Register, Mortality Collection, routine datasets for hospital discharge, and the Cancer Registry, using comparisons with national rates where appropriate. Most importantly, permission will be sought to flag individuals at the Cancer Registry, with access to blood or tissue samples facilitating the identification of genomic 'effect biomarkers'. We hope that the method will be extended to other cohorts and for future deployments.

\section{C.2 LONG TERM PHYSICAL AND MENTAL HEALTH IMPACT OF MILITARY SERVICE}

Malcolm Sim*, Jing Xie, Andrew Forbes, Helen Kelsall. School of Public Health and Preventive Medicine, Monash University, Melbourne, Australia

\subsection{6/OEM-2019-EPI.123}

Background There is emerging recognition of the important co-morbidities and long term relationships between physical and psychological health in military and veteran populations. The aim was to investigate the longitudinal relationships between multisymptom illness (MSI) and psychological disorders.

Methods A cohort of 1990-1991 Gulf War veterans and military comparison group was assessed at Wave 1 (2000-2002) and Wave 2 (2011-2012), including military service characteristics, symptoms, modified Centers for Disease Control (CDC) definition of MSI, the posttraumatic stress disorder (PTSD) Checklist (PCL), and Alcohol Use Disorder Identification Test (AUDIT). The Composite International Diagnostic Interview (CIDI v.2.1) assessed psychological disorders using DSM-IV criteria. Incident cases were defined as participants who did not meet criteria for a health outcome at Wave 1 but met these criteria at Wave 2.

Results Overall participation at Wave 2 was 1390/2779 (50.0\%); 99.8\% completed a health questionnaire including 1356 male participants who were included in these analyses. The adjusted incident rate ratio (IRR) of MSI at Wave 2 was higher for those with, compared to those without, CIDI- 
defined PTSD IRR 3.3 (95\% CI 1.8-6.0), major depression $2.1(1.3-3.5)$ and alcohol disorder $1.8(1.0-3.3)$ at Wave 1. The adjusted IRR of incident CIDI-defined PTSD 3.7 (2.26.3) major depression $2.0(1.2-3.2)$ and AUD $2.1(1.1-3.8)$ at Wave 2 were increased for those with, compared to those without, MSI at Wave 1.

Conclusions Longitudinally, psychological disorders, in particular PTSD and depression, were found to be a risk factor for the development of MSI and the presence of MSI was a risk factor for the development of psychological disorders, in particular PTSD and depression. These findings have important implications for clinical and occupational health practice, service provision and longer term health in military and veteran populations.

\section{C.3 THE MENTAL HEALTH IMPACT OF DEPLOYMENT TO IRAQ AND AFGHANISTAN: WHAT DOES THE CURRENT DATA SHOW?}

\begin{abstract}
${ }^{1}$ Sharon Stevelink*, ${ }^{1}$ Margaret Jones, ${ }^{1}$ Lisa Hull, ${ }^{1}$ David Pernet, ${ }^{1}$ Shirlee MacCrimmon, ${ }^{2}$ Laura Goodwin, ${ }^{1}$ Deirdre MacManus, ${ }^{1,3}$ Dominic Murphy, ${ }^{4}$ Norman Jones, ${ }^{1}$ Neil Greenberg, ${ }^{1}$ Roberto J Rona, ${ }^{1,4}$ Nicola T Fear, ' $S i r$ Simon Wessely. ${ }^{1}$ King's College London, King's Centre for Military Health Research, London, UK; ${ }^{2}$ University of Liverpool, Liverpool, UK; ${ }^{3}$ Combat Stress, London, UK; ${ }^{4}$ Academic Department for Military Mental Health, London, UK
\end{abstract}

\subsection{6/OEM-2019-EPI.124}

The longer term mental health outcomes of UK serving and ex-serving personnel have been the subject of much speculation. The latest research findings from the third phase of a longitudinal study will be presented, which is the only military cohort study of its kind in the UK. Data was collected among 8093 personnel between 2014 and 2016. The study examined the prevalence of mental disorders and alcohol misuse, whether rates differed between serving and ex-serving regular personnel and the impact of various deployment exposures. The prevalence of probable posttraumatic stress disorder was 6.2\% (95\% confidence interval (CI) 5.5-6.9), 21.9\% (95\% CI 20.8-23.0) for common mental disorders and 10.0\% (95\% CI 9.2-10.9) for alcohol misuse. Deployment to Iraq or Afghanistan and self-reported role during deployment were associated with significantly worse mental health outcomes and alcohol misuse in ex-serving regular personnel but not in currently serving regular personnel. There was no association with number of deployments for any outcome. The findings highlight the importance of the continued monitoring of following personnel throughout their military career and beyond. The implications of the study outcomes will be discussed in the light of the mental health provision for serving and ex-serving personnel.

\section{C.4 THE ARMED SERVICES TRAUMA REHABILITATION OUTCOME STUDY (ADVANCE): A COHORT STUDY OF PHYSICAL BATTLEFIELD TRAUMA CASUALTIES}

\footnotetext{
${ }^{1}$ Nicola Fear* ${ }^{*},{ }^{1}$ Daniel Dyball, ${ }^{2}$ Melanie Chesnokov, ${ }^{3}$ Christopher Boos, ${ }^{2}$ Anthony Bull, ${ }^{2}$ Paul Cullinan, ${ }^{4}$ Group Captain Alex Bennett. 'King's College London, London, UK; ${ }^{2}$ Imperial College London, London, UK; ${ }^{3}$ Bournemouth University, Bournemouth, UK; ${ }^{4}$ Ministry of Defence, Loughborough, UK
}

The recent Iraq and Afghanistan conflicts represent a unique time in UK battlefield medicine; with more service personnel surviving complex traumas than any previous time. During these recent conflicts, approximately 830 UK service personnel were very seriously or seriously injured, and over 9000 were aero-medially evacuated. Little is known about the long-term health of these injured personnel. The Armed Services Trauma Rehabilitation Outcome Study (ADVANCE) is a longitudinal cohort study investigating the long-term medical and psychosocial outcomes of physical battlefield trauma casualties. 1200 male subjects who deployed to Afghanistan (600 injured and 600 non-injured individuals' frequencymatched by age, rank, service and role-in-theatre) will be followed up over a 20 year period. Primary objectives are to assess cardiovascular risk, cardiovascular events and osteoarthritis of the hip and knee. Secondary objectives are: musculoskeletal disease; pain; mental health; employment outcomes; functional status; quality of life; relationship status; sexual functioning; lung function; hearing loss and allcause mortality. Data collection started in August 2015 and 705 participants have been recruited (376 non-injured and 329 injured). Most common injuries include single leg amputation, bilateral leg amputation (below knee), genital injuries and shrapnel injuries. Approximately $83 \%$ of participants are from the Army, 14\% from Naval Services (including Royal Marines) and 3\% from the Royal Air Force. The sample overall is comprised of approximately 14\% Officers - with the other $86 \%$ holding lower ranks. Median age of the sample is 33 years at point of recruitment. Baseline data collection is due to be completed by the end of 2019, when follow data collection will commence. There are no other studies of this type currently being conducted and the results will have far reaching implications for the military and veteran community but also for members of the general population who have experienced a severe physical trauma.

\section{C.5 SARIN EXPOSURES IN A COHORT OF BRITISH MILITARY PARTICIPANTS IN HUMAN EXPERIMENTAL RESEARCH AT PORTON DOWN 1945-1987}

${ }^{1}$ Thomas J Keegan*, ${ }^{2}$ Lucy M Carpenter, ${ }^{3}$ Claire Brooks, ${ }^{4}$ Toby Langdon, ${ }^{4}$ Katherine M Venables. 'Lancaster University, Lancaster, UK; ${ }^{2}$ Nuffield College, University of Oxford, Oxford, UK; ${ }^{3}$ Oxford Department of Oncology, Oxford, UK; ${ }^{4}$ Nuffield Department of Population Health, University of Oxford, Oxford, UK

\subsection{6/OEM-2019-EPI.126}

Background The effects of exposure to chemical warfare agents in humans are topical. Porton Down, near Salisbury, is the UK's centre for research on chemical warfare. Since WWI, a programme of experiments involving approximately 30000 participants drawn from the UK armed services has been undertaken there.

Objectives Our aim is to report on exposures to nerve agents, particularly sarin, using detailed exposure data in a cohort of servicemen who attended Porton Down.

Methods We have used existing data on exposures to UK servicemen who attended the human volunteer programme at Porton Down to examine exposures to nerve agents in general and to sarin in particular.

Results Six principal nerve agents were tested on humans, all between 1945 and 1987. Of the 4299 nerve agent tests recorded, $3511(82 \%)$ were with sarin, most commonly in an exposure chamber, with inhalation being the commonest 